\title{
GROUP REPRESENTATIONS IN THE HOMOLOGY OF 3-MANIFOLDS
}

\author{
ALEX BARTEL AND AUREL PAGE
}

\begin{abstract}
If $M$ is a manifold with an action of a group $G$, then the homology group $H_{1}(M, \mathbb{Q})$ is naturally a $\mathbb{Q}[G]$-module, where $\mathbb{Q}[G]$ denotes the rational group ring. We prove that for every finite group $G$, and for every $\mathbb{Q}[G]$-module $V$, there exists a closed hyperbolic 3-manifold $M$ with a free $G$-action such that the $\mathbb{Q}[G]$-module $H_{1}(M, \mathbb{Q})$ is isomorphic to $V$. We give an application to spectral geometry: for every finite set $\mathcal{P}$ of prime numbers, there exist hyperbolic 3-manifolds $N$ and $N^{\prime}$ that are strongly isospectral such that for all $p \in \mathcal{P}$, the $p$-power torsion subgroups of $H_{1}(N, \mathbb{Z})$ and of $H_{1}\left(N^{\prime}, \mathbb{Z}\right)$ have different orders. The main geometric techniques are Dehn surgery and, for the spectral application, the Cheeger-Müller formula, but we also make use of tools from different branches of algebra, most notably of regulator constants, a representation theoretic tool that was originally developed in the context of elliptic curves.
\end{abstract}

\section{INTRODUCTION}

1.1. Group actions on rational homology of 3-manifolds. If $M$ is a manifold with an action by a group $G$, then the homology of $M$ carries a natural $G$-action. The $G$-module structure of integral and rational homology can often be used to deduce information about the manifold, see e.g. [10, 22].

In this paper, we investigate the $G$-module structure of the rational homology of 3-manifolds. In [7] Cooper and Long prove that for every finite group $G$, there exists a hyperbolic rational homology 3 -sphere with a free $G$-action. In fact, their method proves a stronger statement. Let $\mathbb{Q}[G]$ denote the group algebra of $G$ over the field $\mathbb{Q}$ of rational numbers. Cooper and Long define the notion of a canonical $\mathbb{Q}[G]$-module, and prove that every direct sum of canonical $\mathbb{Q}[G]$-modules can be realised as $H_{1}(M, \mathbb{Q})$ for a closed 3-manifold $M$ with a free $G$-action.

In the present paper, we generalise the theorem of Cooper and Long to arbitrary $\mathbb{Q}[G]$-modules. Our main result is the following.

Theorem 1.1. Let $G$ be a finite group, and let $W$ be a finitely generated $\mathbb{Q}[G]$-module. Then there exists a closed connected orientable 3-manifold $M$ with a free orientation preserving $G$-action, such that the $\mathbb{Q}[G]$-module $H_{1}(M, \mathbb{Q})$ is isomorphic to $W$.

Like Cooper and Long, we can also strengthen Theorem 1.1 by ensuring that $M$ is hyperbolic - see Theorem 3.8 .

Remark 1.2. The referees inform us that there is a way of proving Theorem 1.1 by using higher dimensional surgery. The proof we will present here will only use surgery in dimension 3. 
1.2. Application to isospectral manifolds. As an application of Theorem 1.1 we prove a result on torsion homology of isospectral manifolds. This relies on our previous work [2] on the interplay between Sunada's construction of isospectral manifolds [23] and the Cheeger-Müller theorem.

Recall that two Riemannian manifolds $M$ and $M^{\prime}$ are said to be strongly isospectral if the spectra of every natural (i.e. functorially defined) selfadjoint elliptic differential operator on $M$ and $M^{\prime}$ agree. There is a large body of literature devoted to investigating which topological or geometric invariants of manifolds are strongly isospectral invariants, see [21, 12, 11] for surveys. Strongly isospectral Riemannian manifolds necessarily have the same dimension, the same volume, and the same Betti numbers, but for example they may have non-isomorphic real cohomology rings, see [17]. Ikeda has shown that (strongly) isospectral closed 3-manifolds with constant positive curvature are necessarily isometric [13]. In contrast, we show in the present paper that strongly isospectral hyperbolic 3-manifolds can have very different integral homology groups. If $p$ is a prime number, and $A$ is an Abelian group, let $A\left[p^{\infty}\right]$ denote the subgroup of $A$ of elements of $p$-power order.

Theorem 1.3. Let $\mathcal{P}$ be a finite set of prime numbers. Then there exist closed connected orientable 3-manifolds $M$ and $M^{\prime}$ that are strongly isospectral with respect to hyperbolic metrics and such that

(1) for all $p \in \mathcal{P}$ we have

$$
\# H_{1}(M, \mathbb{Z})\left[p^{\infty}\right] \neq \# H_{1}\left(M^{\prime}, \mathbb{Z}\right)\left[p^{\infty}\right] ;
$$

(2) for all prime numbers $q \notin \mathcal{P}$ we have an isomorphism of Abelian groups

$$
H_{1}(M, \mathbb{Z})\left[q^{\infty}\right] \cong H_{1}\left(M^{\prime}, \mathbb{Z}\right)\left[q^{\infty}\right] .
$$

\section{Remarks.}

(1) We will obtain the manifolds $M$ and $M^{\prime}$ in Theorem 1.3 using a construction of Sunada [23], who was guided by a well-known analogy between spectral zeta functions of manifolds and Dedekind zeta functions of number fields.

(2) A weak form of the number theoretic analogue of Theorem 1.3 is an old open problem [8, 4]: do there exist, for every prime number $p$, number fields with the same Dedekind zeta function but with different $p$-class numbers?

(3) Theorem 1.3 certainly does not hold for 2-manifolds, since they have torsion-free homology, while for 4- and higher-dimensional manifolds, Theorem 1.3 has already been known since the work of Sunada. The 3 -dimensional case has been the only open one.

Let $p$ be an odd prime number. Let us briefly explain how to deduce Theorem 1.3 in the special case $\mathcal{P}=\{p\}$ from Theorem 1.1. Consider the following two subgroups of the group $G=\mathrm{GL}_{2}\left(\mathbb{F}_{p}\right)$ of invertible $2 \times 2$ matrices over the finite field with $p$ elements:

$$
B=\left(\begin{array}{cc}
\mathbb{F}_{p}^{\times} & \mathbb{F}_{p} \\
0 & \mathbb{F}_{p}^{\times}
\end{array}\right), \quad H=\left(\begin{array}{cc}
\left(\mathbb{F}_{p}^{\times}\right)^{2} & \mathbb{F}_{p} \\
0 & \mathbb{F}_{p}^{\times}
\end{array}\right) .
$$


We can form the permutation modules $\mathbb{Q}[G / H]$ and $\mathbb{Q}[G / B]$, which are spanned over $\mathbb{Q}$ by the cosets of the respective subgroup, and where $G$ acts by permuting the respective basis. The module $\mathbb{Q}[G / H]$ decomposes as a direct sum $\mathbb{Q}[G / H] \cong \mathbb{Q}[G / B] \oplus I$, where $I$ is a simple $\mathbb{Q}[G]$-module of dimension $(p+1)$ over $\mathbb{Q}$. The first part of Theorem 1.3 for $\mathcal{P}=\{p\}$ immediately follows from Theorem 1.1 and the following result.

Lemma 1.4 ([2], Corollary 4.4). Let $p$ be an odd prime number, let $G=$ $\mathrm{GL}_{2}\left(\mathbb{F}_{p}\right)$, and let $I$ be as above. Suppose that there exists a closed 3 -manifold $X$ with a free $G$-action, such that the multiplicity of $I$ in the $\mathbb{Q}[G]$-module $H_{1}(X, \mathbb{Q})$ is odd. Then there exist closed connected orientable Riemannian 3-manifolds $M$ and $M^{\prime}$ that are strongly isospectral and such that

$$
\# H_{1}(M, \mathbb{Z})\left[p^{\infty}\right] \neq \# H_{1}\left(M^{\prime}, \mathbb{Z}\right)\left[p^{\infty}\right] .
$$

If, in addition, $X$ is hyperbolic, then $M$ and $M^{\prime}$ can be chosen to be hyperbolic.

By inspecting the construction in Lemma 1.4 a bit more closely, one can also deduce the second part of Theorem 1.3 from [2, Theorem 3.5].

For concrete groups $G$ and $\mathbb{Q}[G]$-modules $W$, one can sometimes try to reach the conclusion of Theorem 1.1 by a brute force search. In [2, Proposition 1.5], we were able to prove Theorem 1.3(1) in this way when $\mathcal{P}=\{p\}$ for all $p \leq 71$.

Remark. The canonicity condition on $W$ in the construction of Cooper and Long can be formulated as follows. Let $\mathbb{Q}[G] \mathbb{Q}[G]$ denote the left regular $\mathbb{Q}[G]$-module. Recall that every $\mathbb{Q}[G]$-module can be uniquely written as a direct sum of simple modules. The condition on $W$ for the method of [7] to apply is that for every simple $\mathbb{Q}[G]$-module $V_{i}$, the multiplicity of $V_{i}$ in $W$ be divisible by the multiplicity of $V_{i}$ in the regular module $\mathbb{Q}[G] \mathbb{Q}[G]$. Note that the multiplicity of $I$ in the regular module of $\mathrm{GL}_{2}\left(\mathbb{F}_{p}\right)$ is $p+1$, so if $W$ is a canonical $\mathbb{Q}\left[\mathrm{GL}_{2}\left(\mathbb{F}_{p}\right)\right]$-module, then the multiplicity of $I$ in $W$ is even. In particular, the result of Cooper-Long is not sufficient to apply Lemma 1.4 .

The proof of the general case of Theorem 1.3 will involve the same ideas as that of the special case sketched above, but will require more algebraic preparation, and will occupy Section 5 .

1.3. Ingredients of the proof. The proof of the main theorem will be given in Section 3. We will show how, given a 3-manifold $M$ with a free $G$ action, and a finitely generated $\mathbb{Q}[G]$-module $V$, one may perform a sequence of $G$-equivariant Dehn surgeries to produce a 3-manifold $M^{\prime}$ with a free $G$ action such that there is an isomorphism of $\mathbb{Q}[G]$-modules $H_{1}\left(M^{\prime}, \mathbb{Q}\right) \cong$ $H_{1}(M, \mathbb{Q}) \oplus V$ - see Corollary 3.7; and also how, given a 3-manifold $M$ with a free $G$-action such that $H_{1}(M, \mathbb{Q})$ has a $\mathbb{Q}[G]$-submodule isomorphic to $\mathbb{Q}[G] \mathbb{Q}[G]$, one can "kill" that submodule - see Proposition 3.5. Starting from a 3-manifold with a free $G$-action and arbitrary homology, one can then iterate the above two steps to realise any given $\mathbb{Q}[G]$-module - see Theorem 3.8.

One of these surgeries is prescribed by the coefficients of an idempotent $e \in \mathbb{Q}[G]$ such that $\mathbb{Q}[G] e \cong V$, and for this step to yield the desired result, 
we need $e$ to satisfy a technical algebraic condition. The following result, which we will prove in Section 2 as Corollary 2.10, says that all idempotents in $\mathbb{Q}[G]$ indeed do possess the required property.

Proposition 1.5. Let $G$ be a finite group. Given an element $x=\sum_{g \in G} a_{g} g$ of $\mathbb{Q}[G]$, where $a_{g} \in \mathbb{Q}$, define $x^{*}=\sum_{g \in G} a_{g} g^{-1} \in \mathbb{Q}[G]$. Then for every idempotent e $\in \mathbb{Q}[G]$, we have $\mathbb{Q}[G] \mathbb{Q}[G]=\mathbb{Q}[G] e+\mathbb{Q}[G]\left(1-e^{*}\right)$.

Note that if the star is dropped, then the conclusion immediately follows from the definition of an idempotent. On the other hand, since $e$ is not assumed to be central, one does not, in general, have $e=e^{*}$. Moreover, if the operator $x \mapsto x^{*}$ is replaced by a different involution (see Section 2.1) on $\mathbb{Q}[G]$, then there may not exist an idempotent with the required properties at all. It is important to note that Proposition 1.5 does not follow from the well-known fact that $\mathbb{Q}[G] e^{*}$ is abstractly isomorphic to $\mathbb{Q}[G] e$; see in particular Example 2.8.

1.4. Generalisations. The main geometric step, in which we add a given $\mathbb{Q}[G]$-module to the homology of a given 3-manifold with a free $G$-action actually works in greater generality. For example, instead of a free $G$-action we may allow an orientation preserving action by isometries with no element acting trivially, which implies that the fixed point set under every $g \in G$ is at most 1-dimensional. For the precise statements, see Theorems 4.1 and 4.2 .

This has the somewhat surprising consequence that given a closed connected orientable Riemannian 3-manifold $M$ with an orientation preserving effective $G$-action, one can infer no information about the fixed points from the structure of $H_{1}(M, \mathbb{Q})$ : if a certain configuration of fixed point spaces can be realised at all, then it can be realised with $H_{1}(M, \mathbb{Q})$ being isomorphic to any given $\mathbb{Q}[G]$-module. This stands in stark contrast to the situation in dimension 2, as we point out in Section 4. See in particular Corollary 4.6, which, vaguely speaking, says that for closed connected orientable surfaces $M$ with a $G$-action, $H_{1}(M, \mathbb{Q})$ "knows" everything about the fixed point structure.

In this paper, when we say "manifold", we will always mean a closed connected oriented smooth manifold, all automorphisms will be orientation preserving, and all maps between manifolds will be smooth.

Acknowledgements. We are grateful to Nathan Dunfield for making us aware of the paper of Cooper and Long, and for his incredibly prompt and helpful responses to our questions, to Allan Edmonds for many helpful remarks concerning the state of knowledge on the subject and the exposition, to John Smillie and Karen Vogtmann for helpful discussions, and to Hendrik Lenstra for giving the proof of Proposition 1.5 that we present here, which is much simpler than the proof we gave in an earlier draft. We are also very grateful to an anonymous referee for helpful comments that greatly improved the exposition! Most of this work was done while the authors were at the University of Warwick. We thank the Mathematics department at Warwick for a very stimulating working environment. Finally, we thank the EPSRC for financial support via a First Grant, respectively the EPSRC Programme Grant EP/K034383/1 LMF: L-Functions and Modular Forms. 


\section{Algebras with an involution}

In this section, we will prove Proposition 1.5 .

2.1. Semisimple algebras. The main reference for this subsection is [16]. All our rings are associative, and have a unit element, denoted by 1 . All our modules are left modules, and are assumed to be finitely generated. If $R$ is a ring, then $\mathrm{Z}(R)$ denotes the centre of $R$; the regular module ${ }_{R} R$ is defined as having the same underlying additive group as $R$, and the $R$-action being given by left multiplication.

Let $K$ be a field. A $K$-algebra is a ring $A$ that is equipped with a ring homomorphism $K \rightarrow \mathrm{Z}(A)$. All our $K$-algebras are finite-dimensional over $K$. If $A$ is a $K$-algebra, then the trace $\operatorname{Tr}_{A / K}(a)$ of an element $a \in A$ is defined to be the trace of the endomorphism of the $K$-vector space $A$ given by multiplication by $a$ on the left.

Example 2.1. Let $A=\mathbb{Q}[G]$ be the group algebra of $G$ over $\mathbb{Q}$, and let $a=\sum_{g \in G} a_{g} g$ be an arbitrary element of $A$. Then $\operatorname{Tr}_{A / \mathbb{Q}} a=\operatorname{dim}_{\mathbb{Q}} A \cdot a_{1}=$ $\# G \cdot a_{1}$.

If $A$ is a $K$-algebra, then an $A$-module $V$ is called simple if it has exactly two submodules, 0 and $V$; a simple submodule of ${ }_{A} A$ is the same thing as a minimal left ideal of $A$. The Jacobson radical of a $K$-algebra $A$ is the set of elements $a \in A$ that annihilate every simple $A$-module; it is a two-sided ideal of $A$. A $K$-algebra $A$ is called semisimple if its Jacobson radical is 0 . For every integer $n \geqslant 1$, let $\mathrm{M}_{n}(K)$ be the $K$-algebra of $n \times n$ matrices over $K$. We will use the following basic result.

Lemma 2.2. Let $K$ be a field and let $A$ be a semisimple $K$-algebra. Then there exists a finite field extension $L / K$ such that $L \otimes_{K} A$ is isomorphic to a product of algebras of the form $\mathrm{M}_{n}(L)$ for integers $n \geqslant 1$.

An idempotent in an algebra $A$ is an element $e \in A$ such that $e^{2}=e$. If $e \in$ $A$ is an idempotent, then so is $1-e$, and in this case one has a decomposition into left ideals $A=A e \oplus A(1-e)$. If an algebra $A$ is semisimple, then every simple $A$-module is isomorphic to some minimal left ideal of $A$, every $A$ module is a direct sum of simple submodules, and for every left ideal $I$ in $A$, there exists an idempotent $e$ in $A$ such that $I=A e$.

An anti-automorphism of an algebra $A$ is a $K$-linear automorphism $\alpha: A \rightarrow$ $A$ such that $\alpha(1)=1$ and $\alpha(x y)=\alpha(y) \alpha(x)$ for all $x, y \in A$. An involution on $A$ is an anti-automorphism $\iota$ such that $\iota \circ \iota=\mathrm{id}$.

Let $V$ be a finite-dimensional vector space over $K$, equipped with a symmetric bilinear form $\phi: V \times V \rightarrow K$. If $X \subset V$ is a subset, then its orthogonal complement is defined to be

$$
X^{\perp}=\{v \in V \mid \phi(v, x)=0 \text { for all } x \in X\} .
$$

The bilinear form $\phi$ is called non-degenerate if $V^{\perp}=0$, and it is called anisotropic if for every nonzero $v \in V$ we have $\phi(v, v) \neq 0$. Note that $\phi$ is non-degenerate if and only if the induced map $V \rightarrow \operatorname{Hom}(V, K)$ given by $v \mapsto(w \mapsto \phi(v, w))$ is an isomorphism. It follows that if $\phi$ is nondegenerate, then for every subspace $W \subset V$, we have, by the rank-nullity 
formula, $\operatorname{dim} V=\operatorname{dim} W+\operatorname{dim} W^{\perp}$. If $\phi$ is anisotropic, then it is nondegenerate, and for every subspace $W \subset V$ we have $V=W+W^{\perp}$.

Lemma 2.3. Let $A$ be a semisimple $K$-algebra, and let $\iota$ be an involution on $A$. Then for all $x \in A$ we have $\operatorname{Tr}_{A / K}(x)=\operatorname{Tr}_{A / K}(\iota(x))$. In particular, the $K$-bilinear form $(x, y) \mapsto \operatorname{Tr}_{A / K}(x \iota(y))$ on $A$ is symmetric.

Proof. See [20, 13.1 (iv)].

Remark 2.4. In Lemma 2.3 the semisimplicity assumption is necessary: let $A$ be the $K$-algebra of upper-triangular $2 \times 2$ matrices with coefficients in $K$, equipped with the involution

$$
\iota:\left(\begin{array}{ll}
a & b \\
0 & d
\end{array}\right) \mapsto\left(\begin{array}{cc}
d & -b \\
0 & a
\end{array}\right) .
$$

Then $\operatorname{Tr}_{A / K}\left(\begin{array}{ll}a & b \\ 0 & d\end{array}\right)=2 a+d$, which is not preserved by $\iota$.

Let $A$ be a semisimple $K$-algebra and $\iota$ be an involution on $A$. The associated symmetric bilinear form on $A$ is

$$
\phi_{\iota}:(x, y) \mapsto \operatorname{Tr}_{A / K}(x \iota(y)) .
$$

We say that $\iota$ is non-degenerate (resp. anisotropic) if $\phi_{\iota}$ is non-degenerate (resp. anisotropic).

2.2. Idempotents and anisotropic involutions. In this subsection we prove the main algebraic result, Proposition 2.7. The proof we give here was communicated to us by Hendrik Lenstra, and is much simpler than the proof we gave in an earlier draft of the paper.

Lemma 2.5. Let $A$ be a semisimple $K$-algebra. Then for all $x \in A$ we have $\operatorname{dim}_{K} A x=\operatorname{dim}_{K} x A$.

Proof. The result is true if $A$ is a product of matrix algebras over $K$. Let $A$ be an arbitrary semisimple $K$-algebra. If $L / K$ is a finite field extension, then we have $\operatorname{dim}_{L}\left(L \otimes_{K} A\right) x=\operatorname{dim}_{K} A x$, and similarly for $x A$. The general case of the lemma therefore follows from the special case and Lemma 2.2.

Note that if $A$ is a $K$-algebra with an involution $\iota$, and $e \in A$ is an idempotent, then $\iota(e)$ is also an idempotent.

Lemma 2.6. Let $A$ be a semisimple $K$-algebra with a non-degenerate involution $\iota$. Then for every idempotent $e \in A$ we have $(A e)^{\perp}=A(1-\iota(e))$, where the orthogonal complement is taken with respect to $\phi_{\iota}$.

Proof. Since $e$ is idempotent, we have $A(1-\iota(e)) \subset(A e)^{\perp}$. On the other hand we have

$$
\begin{aligned}
\operatorname{dim}(A e)^{\perp} & =\operatorname{dim} A-\operatorname{dim} A e \\
& =\operatorname{dim} A(1-e) \\
& =\operatorname{dim}(1-e) A \\
& =\operatorname{dim} A(1-\iota(e))
\end{aligned}
$$

where the four equalities follow, respectively, from the assumption that $\iota$ is non-degenerate, from the assumption that $e$ is an idempotent, from 
Lemma 2.5, and from the assumption that $\iota$ is an anti-automorphism. The claimed equality follows.

We now prove the main result of the section.

Proposition 2.7. Let $A$ be a semisimple $\mathbb{Q}$-algebra with an anisotropic involution $\iota$. Then for every idempotent $e \in A$, we have ${ }_{A} A=A e+A(1-$ $\iota(e))$.

Proof. By Lemma 2.6 we have $(A e)^{\perp}=A(1-\iota(e))$. Since $\iota$ is anisotropic, we have $A=A e+(A e)^{\perp}$, giving the result.

Example 2.8. Proposition 2.7 is false without the anisotropy assumption, even if the algebra is simple. For instance, the split quaternion algebra $A=\mathrm{M}_{2}(\mathbb{Q})$, the involution

$$
\left(\begin{array}{ll}
a & b \\
c & d
\end{array}\right) \mapsto\left(\begin{array}{cc}
d & -b \\
-c & a
\end{array}\right)
$$

and the idempotent $e=\left(\begin{array}{ll}1 & 0 \\ 0 & 0\end{array}\right)$ provide a counter-example. This example shows, in particular, that Proposition 2.7 is not a formal consequence of the fact that $A e$ is isomorphic to $A \iota(e)$ as $A$-modules.

Definition 2.9. Let $G$ be a finite group. Recall that the group algebra $\mathbb{Q}[G]$ is a semisimple $\mathbb{Q}$-algebra. Define an involution $x \mapsto x^{*}$ on $\mathbb{Q}[G]$ by setting $g^{*}=g^{-1}$ for all $g \in G$, and extending $\mathbb{Q}$-linearly.

Corollary 2.10. Let $G$ be a finite group. Then for every idempotent $e \in$ $\mathbb{Q}[G]$, we have $\mathbb{Q}[G] \mathbb{Q}[G]=\mathbb{Q}[G] e+\mathbb{Q}[G]\left(1-e^{*}\right)$.

Proof. If $a=\sum_{g \in G} a_{g} g$ is an arbitrary element of $\mathbb{Q}[G]$, then the coefficient of the identity $1 \in G$ in $a a^{*}$ is $\sum_{g \in G} a_{g}^{2}$. It therefore follows from Example 2.1 that the involution $x \mapsto x^{*}$ is anisotropic. The result follows from Proposition 2.7, applied to $A=\mathbb{Q}[G]$ and $\iota=\left(x \mapsto x^{*}\right)$.

\section{Proof OF THE MAIN THEOREM}

In this section, we prove Theorem 3.8, which is a strengthening of Theorem 1.1 from the introduction. Let $G$ be a finite group. Our proof will proceed by a sequence of Dehn surgeries on a 3-manifold with a $G$-action.

Definitions 3.1. (1) Let $M$ be a manifold with an action of $G$. We say that a subset $C \subseteq M$ is $G$-disjoint if for every $g \in G \backslash\{1\}$, the intersection $C \cap g C$ is empty, equivalently if the restriction to $C$ of the covering map $M \rightarrow M / G$ is injective.

(2) Below, the manifolds $S^{1}$ and $\partial D^{2}$ are understood to be oriented. If $M$ is a 3-manifold with a $G$-action, and $\varphi: S^{1} \times D^{2} \rightarrow M$ is an embedding with a $G$-disjoint image, let $M(\varphi)=M \backslash G \cdot \varphi$ (interior of $S^{1} \times D^{2}$ ). Let $\gamma, \gamma^{\prime}$ be simple closed curves on $\varphi\left(S^{1} \times \partial D^{2}\right)$ whose classes in the fundamental group $\pi_{1}\left(\varphi\left(S^{1} \times \partial D^{2}\right)\right)$ together generate that fundamental group. Then the result of $G$-equivariant surgery on $M$ along $\varphi, \gamma$ is the manifold $M(\varphi, \gamma)$ defined as $M(\varphi) \sqcup \bigsqcup_{g \in G} g\left(S^{1} \times D^{2}\right)$, where each $g\left(S^{1} \times D^{2}\right)$ is a copy of $S^{1} \times D^{2}$, with the obvious $G$-action on the disjoint union, modulo the equivalence relation that identifies the boundary of 
$M(\varphi)$ with that of $\bigsqcup_{g \in G} g\left(S^{1} \times D^{2}\right)$ by identifying, for all $g \in G$, the curve $g \gamma$ with the simple closed curve $g\left(\{1\} \times \partial D^{2}\right) \subset g\left(S^{1} \times \partial D^{2}\right)$, and $g \gamma^{\prime}$ with the simple closed curve $g\left(S^{1} \times\{1\}\right) \subset g\left(S^{1} \times \partial D^{2}\right)$. The diffeomorphism class of $M(\varphi, \gamma)$ does not depend on the choice of $\gamma^{\prime}$.

If $M$ is a 3 -manifold, then we denote the intersection pairing $H_{2}(M, \mathbb{Z}) \times$ $H_{1}(M, \mathbb{Z}) \rightarrow \mathbb{Z}$ by $(x, y) \mapsto x \cdot y$, and we use the same notation for $\mathbb{Q}$ coefficients in place of $\mathbb{Z}$.

We will make repeated use of the following variant of [15, Lemma 5.6].

Lemma 3.2. Let $M$ be a 3-manifold, let $\varphi: S^{1} \times D^{2} \rightarrow M$ be an embedding with a $G$-disjoint image, and let $\gamma$ be a simple closed curve on $\varphi\left(S^{1} \times \partial D^{2}\right)$. Then the row and the column in the diagram

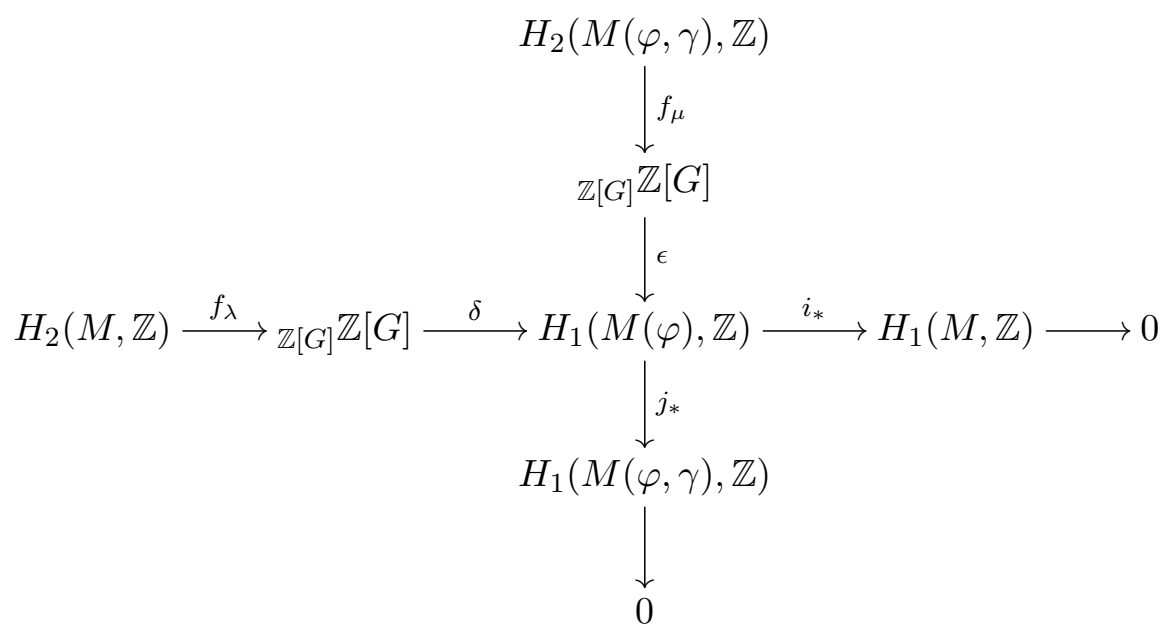

of $\mathbb{Z}[G]$-modules are exact, where the maps are defined as follows:

- $i_{*}$ and $j_{*}$ are induced by the canonical injections of $M(\varphi)$ into $M$, respectively $M(\varphi, \gamma)$;

- $\delta$ sends 1 to the class of $\varphi\left(\{1\} \times \partial D^{2}\right)$, and $\epsilon$ sends 1 to the class of $\gamma$;

- $f_{\lambda}=\left(x \mapsto \sum_{g \in G}(x \cdot g \lambda) g\right)$, where $\lambda$ is the curve $\varphi\left(S^{1} \times\{0\}\right) \subset M$, and $f_{\mu}=\left(x \mapsto \sum_{g \in G}(x \cdot g \mu) g\right)$, where $\mu$ is the curve $S^{1} \times\{0\} \subset S^{1} \times D^{2} \subset$ $M(\varphi, \gamma)$.

Proof. The proof is identical to that of [15, Lemma 5.6].

Below we will also use the notation of Lemma 3.2 for homology with $\mathbb{Q}$ coefficients. The two basic Dehn surgeries that we will use in the proof of Theorem 3.8 are described in Lemma 3.3 and Proposition 3.5 .

Lemma 3.3. Let $M$ be a 3-manifold with a free $G$-action. Then there exists a 3-manifold $M^{\prime}$ with a free $G$-action such that we have an isomorphism $H_{1}\left(M^{\prime}, \mathbb{Q}\right) \cong H_{1}(M, \mathbb{Q}) \oplus \mathbb{Q}[G] \mathbb{Q}[G]$ of $\mathbb{Q}[G]$-modules.

Proof. Let $\varphi: S^{1} \times D^{2} \rightarrow M$ be a $G$-disjoint embedding that sends $S^{1} \times\{1\}$ to a null-homotopic simple closed curve $\gamma$ in $M \backslash G \cdot \varphi\left(\right.$ interior of $\left.S^{1} \times D^{2}\right)$, and let $M^{\prime}=M(\varphi, \gamma)$. Then, $\lambda$ is also null-homotopic in $M$, so the map $f_{\lambda}$ of Lemma 3.2 is the zero map, so that the map $\delta$ of the lemma is injective; and also, since $\gamma$ is null-homotopic, the map $\epsilon$ of Lemma 3.2 is the zero 
map, so that the map $j_{*}$ of the lemma is injective. By Lemma 3.2, the manifold $M^{\prime}$ has the required property.

Recall from Definition 2.9 the involution on $\mathbb{Q}[G]$ given by $g \mapsto g^{*}=g^{-1}$ for all $g \in G$.

Lemma 3.4. Let $G$ be a finite group, let $e \in \mathbb{Q}[G]$ be an idempotent, and let $y \in \mathbb{Q}[G]$ be arbitrary. Let $A=\mathbb{Q}[G]$ e, and for $s \in \mathbb{Q}$ let $B(s)=\{b \in$ $\left.\mathbb{Q}[G]: b(1+s y) \in \mathbb{Q}[G]\left(1-e^{*}\right)\right\}$. Then for all but finitely many $s \in \mathbb{Q}$, we have $A \cap B(s)=\{0\}$.

Proof. For all but finitely many $s \in \mathbb{Q}$, the element $1+s y$ is invertible, since the multiplication-by-y map on $\mathbb{Q}[G]$ has only finitely many eigenvalues. This implies that for all but finitely many $s \in \mathbb{Q}$, the $\mathbb{Q}$-vector subspace $B(s)$ of $\mathbb{Q}[G]$ has dimension $\operatorname{dim} \mathbb{Q}[G]\left(1-e^{*}\right)=\operatorname{dim} \mathbb{Q}[G](1-e)=\operatorname{dim} \mathbb{Q}[G]-$ $\operatorname{dim} A$. We deduce that for all but finitely many $s \in \mathbb{Q}$, the condition $A \cap$ $B(s)=\{0\}$ is equivalent to $\operatorname{dim}(A+B(s))=\operatorname{dim} \mathbb{Q}[G]$, which is equivalent to the non-vanishing of a determinant that is a polynomial in $s$. Since $B(0)=\mathbb{Q}[G]\left(1-e^{*}\right)$, Corollary 2.10 implies that $A+B(0)=\mathbb{Q}[G]$, so the above determinant is not identically 0 , so has only finitely many roots, as claimed.

Proposition 3.5. Let $P$ be a submodule of $\mathbb{Q}[G] \mathbb{Q}[G]$, let $U$ be a $\mathbb{Q}[G]$ module, and let $M$ be a 3-manifold with a free $G$-action such that there is an isomorphism $H_{1}(M, \mathbb{Q}) \cong \mathbb{Q}[G] \mathbb{Q}[G] \oplus U$ of $\mathbb{Q}[G]$-modules. Then there exists a 3-manifold $M^{\prime}$ with a free $G$-action such that there is an isomorphism $H_{1}\left(M^{\prime}, \mathbb{Q}\right) \cong P \oplus U$ of $\mathbb{Q}[G]$-modules.

Proof. Let $l \in H_{1}(M, \mathbb{Z})$ be such that $\mathbb{Q}[G] l$ is the direct summand $\mathbb{Q}[G] l \cong$ $\mathbb{Q}[G] \mathbb{Q}[G]$ of $H_{1}(M, \mathbb{Q})$, let $e \in \mathbb{Q}[G]$ be an idempotent such that we have an isomorphism $\mathbb{Q}[G] e \cong P$ of $\mathbb{Q}[G]$-modules, let $d \in \mathbb{Z}_{>0}$ be such that de $\in \mathbb{Z}[G]$, let $\lambda$ be a simple closed curve in $M$ representing the class $[\lambda]=$ $d(1-e) l \in H_{1}(M, \mathbb{Z})$, and let $\varphi: S^{1} \times D^{2} \rightarrow M$ be a $G$-disjoint embedding such that $\varphi\left(S^{1} \times\{0\}\right)=\lambda$.

First, we claim that, with the above choices, the kernel of the map $\delta$ of Lemma 3.2 is $\mathbb{Q}[G]\left(1-e^{*}\right)$, so that $H_{1}(M(\varphi), \mathbb{Q}) \cong H_{1}(M, \mathbb{Q}) \oplus \mathbb{Q}[G] e^{*} \cong$ $H_{1}(M, \mathbb{Q}) \oplus P$, with the summand $P$ being generated by the class of $\varphi(\{1\} \times$ $\left.\partial D^{2}\right)$. To prove the claim, let $x \in H_{2}(M, \mathbb{Q})$ have intersection number 1 with $l$, and intersection number 0 with $g l$ for all $g \in G \backslash\{1\}$ and with all classes in $U$. Such an element exists by Poincaré duality. Then it is clear that the image of $f_{\lambda}$ is generated, as a $\mathbb{Q}[G]$-module, by $f_{\lambda}(x)$. Write $d(1-e)=\sum_{h \in G} a_{h} h$, where $a_{h} \in \mathbb{Z}$ for all $h \in G$. For every $g \in G$, the intersection number $x \cdot g \lambda$ is the coefficient in $g d(1-e)$ of the identity, which is $a_{g^{-1}}$, so that $f_{\lambda}(x)=\sum_{g \in G}(x \cdot g \lambda) g=\sum_{g \in G} a_{g^{-1}} g=d\left(1-e^{*}\right)$, as claimed.

Let $\mathcal{M}$ be the class in $H_{1}(M(\varphi), \mathbb{Q})$ of the simple closed curve $\varphi(\{1\} \times$ $\left.\partial D^{2}\right) \subset \varphi\left(S^{1} \times \partial D^{2}\right)$, and let $\mathcal{L}$ be the class of a simple closed curve on $\varphi\left(S^{1} \times \partial D^{2}\right)$ such that $i_{*}(\mathcal{L})=[\lambda]$.

Our second claim is that for all but finitely many values of $q / p$, where $p$ and $q$ are coprime integers, if $\gamma$ is a simple closed curve on $\varphi\left(S^{1} \times \partial D^{2}\right)$ whose class $[\gamma]$ in $H_{1}(M(\varphi), \mathbb{Q})$ is $p \mathcal{M}+q \mathcal{L}$, then the map $\epsilon$ of Lemma 3.2 is injective. Indeed, let $p$ and $q$ be coprime integers, let $\gamma$ be as just described, 
and suppose that $a \in \mathbb{Q}[G] \mathbb{Q}[G]$ is such that $\epsilon(a)=a[\gamma]=0$. Let $C$ be a $\mathbb{Q}[G]$ submodule of $H_{1}(M(\varphi), \mathbb{Q})$ such that there is a direct sum decomposition of $\mathbb{Q}[G]$-modules

$$
H_{1}(M(\varphi), \mathbb{Q})=\delta(\mathbb{Q}[G] \mathbb{Q}[G]) \oplus C=\mathbb{Q}[G] \mathcal{M} \oplus C .
$$

Then we may write $\mathcal{L}=y \mathcal{M}+c$, where $y \in \mathbb{Q}[G]$ and $c \in C$, so that $a[\gamma]=(a p+a q y) \mathcal{M}+a q c$. By the direct sum decomposition (3.6), the assumption that $a[\gamma]=0$ is equivalent to

(i) $(a p+a q y) \mathcal{M}=0$ and

(ii) $a q c=0$.

Since $\mathbb{Q}[G] \mathcal{M}$ is precisely the kernel of $i_{*}$, condition (ii) is equivalent to $0=i_{*}(a q c)=i_{*}(a q \mathcal{L})=a q[\lambda]=a q d(1-e) l \in H_{1}(M, \mathbb{Q})$, which, for $q \neq 0$, is equivalent to $a$ being contained in the left annihilator $A$ of $1-e$. That annihilator is equal to $\mathbb{Q}[G]$ e. Condition (i), on the other hand, is equivalent to $0=(a p+a q y) \mathcal{M}=\delta(a p+a q y)$, in other words to $a$ being contained in $B\left(\frac{q}{p}\right)=\left\{b \in \mathbb{Q}[G]: b\left(1+\frac{q}{p} y\right) \in \operatorname{ker} \delta\right\}$. Since $\operatorname{ker} \delta=\mathbb{Q}[G]\left(1-e^{*}\right)$, Lemma 3.4 implies that for all but finitely many values of $q / p$, we have $A \cap B\left(\frac{q}{p}\right)=\{0\}$, which proves the second claim.

It immediately follows from the two claims and Lemma 3.2 that for all but finitely many values of $q / p$, we have

$$
H_{1}(M(\varphi, \gamma), \mathbb{Q}) \oplus \mathbb{Q}[G] \mathbb{Q}[G] \cong H_{1}(M(\varphi), \mathbb{Q}) \cong H_{1}(M, \mathbb{Q}) \oplus P,
$$

so that $M^{\prime}=M(\varphi, \gamma)$ satisfies the conclusion of the proposition.

Corollary 3.7. Let $M_{0}$ be a 3-manifold with a free $G$-action, and let $V$ be $a \mathbb{Q}[G]$-module. Then there exists a 3-manifold $M$ with a free $G$-action such that $H_{1}(M, \mathbb{Q}) \cong H_{1}\left(M_{0}, \mathbb{Q}\right) \oplus V$.

Proof. Let $V \cong \bigoplus_{i=1}^{r} P_{i}$, where each $P_{i}$ is a submodule of $\mathbb{Q}[G] \mathbb{Q}[G]$. Define the 3-manifolds $M_{i}$ and $M_{i}^{\prime}$ inductively as follows: supposing that $M_{i-1}$ has been defined, by Lemma 3.3 there exists a 3 -manifold $M_{i}^{\prime}$ with a free $G$-action such that $H_{1}\left(M_{i}^{\prime}, \mathbb{Q}\right) \cong H_{1}\left(M_{i-1}, \mathbb{Q}\right) \oplus \mathbb{Q}[G] \mathbb{Q}[G]$, and by Proposition 3.5 applied to the manifold $M_{i}^{\prime}$ and the $\mathbb{Q}[G]$-module $P_{i}$, there exists a 3 -manifold $M_{i}$ with a free $G$-action such that $H_{1}\left(M_{i}, \mathbb{Q}\right) \cong H_{1}\left(M_{i-1}, \mathbb{Q}\right) \oplus P_{i}$. The manifold $M=M_{r}$ then satisfies the conclusions of the corollary.

By a hyperbolic manifold we mean a connected oriented smooth manifold whose interior is equipped with a Riemannian metric with constant curvature -1 . We can now deduce the main theorem, which is stronger than Theorem 1.1 and which reads as follows.

Theorem 3.8. Let $G$ be a finite group, and let $W$ be a finitely generated $\mathbb{Q}[G]$-module. Then there exists a closed hyperbolic 3-manifold $M^{\prime}$ with a free $G$-action such that the $\mathbb{Q}[G]$-module $H_{1}\left(M^{\prime}, \mathbb{Q}\right)$ is isomorphic to $W$.

Proof. Let $M_{0}$ be a 3-manifold with a free $G$-action. There are many constructions of such manifolds, see e.g. [7, §1]. We can apply Corollary 3.7 to obtain a 3-manifold $M_{1}$ with a free $G$-action such that for some integer $n \geq 1$, there exists an isomorphism $H_{1}\left(M_{1}, \mathbb{Q}\right) \cong \mathbb{Q}[G] \mathbb{Q}[G]^{\oplus n} \oplus W$ of $\mathbb{Q}[G]$-modules. By repeated application of Proposition 3.5 with $P=\{0\}$, 
we may obtain a 3 -manifold $M_{2}$ with a free $G$-action such that there is an isomorphism of $\mathbb{Q}[G]$-modules $H_{1}\left(M_{2}, \mathbb{Q}\right) \cong W$.

We now follow the argument of [7, Theorem 2.6] to obtain a hyperbolic such manifold. Let $p: M_{2} \rightarrow M_{2} / G$ be the covering map. By [3, Proposition 4.2], the manifold $M_{2} / G$ contains a null-homotopic simple closed curve $k$ such that $\left(M_{2} / G\right) \backslash k$ is a complete hyperbolic manifold with a single cusp and such that $p^{-1}(k)$ is a union of $\# G$ simple closed curves that bound disjoint discs in $M_{2}$ (see also the first paragraph of [3, Proof of Lemma 4.3]). Let $\varphi: S^{1} \times D^{2} \rightarrow M_{2}$ be an embedding with a $G$-disjoint image such that $\varphi\left(S^{1} \times\{0\}\right)$ is one of these simple closed curves. By [3, Lemma 4.3], for all but one slope $\gamma$ on $\varphi\left(S^{1} \times \partial D^{2}\right)$, the $G$-equivariant surgery along $\varphi, \gamma$ on $M_{2}$ yields a closed manifold $M_{2}(\varphi, \gamma)$ with a free $G$-action, satisfying $H_{1}\left(M_{2}(\varphi, \gamma), \mathbb{Q}\right) \cong W$. By Thurston's hyperbolic Dehn surgery theorem [24, Theorem 5.8.2], equivariant surgery for all but finitely many of these slopes results in a hyperbolic manifold $M^{\prime}$.

Remark 3.9. The last paragraph of the above proof can be replaced by an appeal to Theorem A in the very recent preprint [1].

\section{Homology and the structure of Fixed point Sets}

In this section, we first briefly discuss the analogues of the results in Section 3 for $G$-actions that are not necessarily free. We will omit most details, since the proofs are essentially identical to those of Section 3 . We then compare these results to the very different situation of group actions on 2-dimensional manifolds.

The proof of Lemma 3.3 goes through in the following greater generality: we may allow $M$ to have a $G$-stable "bad region" $M^{\text {bad }} \subseteq M$ that is allowed to be an orbifold, and in which non-trivial elements of $G$ are allowed to have fixed points. This set will then be avoided during the sequence of surgeries. Moreover, the proof of Proposition 3.5 also goes through in that generality, as long as the summand $\mathbb{Q}[G] \mathbb{Q}[G]$ of $H_{1}(M, \mathbb{Q})$ is contained in the image of the natural map $H_{1}\left(M \backslash M^{\text {bad }}, \mathbb{Q}\right) \rightarrow H_{1}(M, \mathbb{Q})$. One therefore deduces the following generalisations of Corollary 3.7 and of Theorem 3.8. In the next two results, let $\mathcal{C}$ be the category of connected topological 3-dimensional orbifolds, possibly with boundary, and let $\mathcal{C}^{\prime}$ be the full subcategory of $\mathcal{C}$ whose objects are oriented manifolds without boundary. All group actions will be assumed to be by homeomorphisms.

Theorem 4.1. Let $M_{0} \in \mathcal{C}$, with an action of a finite group $G$, and let $V$ be a finitely generated $\mathbb{Q}[G]$-module. Let $M_{0}^{\text {bad }} \subseteq M_{0}$ be a subset that satisfies the following conditions:

(a) $M_{0}^{\mathrm{bad}}$ is G-stable;

(b) the complement $M_{0} \backslash M_{0}^{\text {bad }}$ is in $\mathcal{C}^{\prime}$;

(c) the group $G$ acts freely on $M_{0} \backslash M_{0}^{\text {bad }}$ by orientation preserving automorphisms.

Then there exists $M \in \mathcal{C}$ with a $G$-action, and a $G$-stable subset $M^{\text {bad }} \subseteq M$ such that

(1) the complement $M \backslash M^{\text {bad }}$ is in $\mathcal{C}^{\prime}$, and $G$ acts freely by orientation preserving automorphisms on it, 
(2) there is a G-equivariant homeomorphism from $M_{0}^{\mathrm{bad}}$ to $M^{\mathrm{bad}}$,

(3) there is an isomorphism of $\mathbb{Q}[G]$-modules $H_{1}(M, \mathbb{Q}) \cong H_{1}\left(M_{0}, \mathbb{Q}\right) \oplus V$.

Theorem 4.2. Let $M_{0} \in \mathcal{C}$ be such that $H_{1}\left(M_{0}, \mathbb{Q}\right)$ is finite dimensional over $\mathbb{Q}$, with an action of a finite group $G$, and let $W$ be a finitely generated $\mathbb{Q}[G]$-module. Let $M_{0}^{\text {bad }} \subseteq M_{0}$ be a subset that satisfies the following conditions:

(a) $M_{0}^{\mathrm{bad}}$ is $G$-stable;

(b) the complement $M_{0} \backslash M_{0}^{\text {bad }}$ is in $\mathcal{C}^{\prime}$;

(c) the group $G$ acts on $M_{0} \backslash M_{0}^{\text {bad }}$ freely by orientation preserving automorphisms;

(d) the canonical map $H_{1}\left(M_{0} \backslash M_{0}^{\text {bad }}, \mathbb{Q}\right) \rightarrow H_{1}\left(M_{0}, \mathbb{Q}\right)$ is surjective.

Then there exists $M \in \mathcal{C}$ with a $G$-action, and a subset $M^{\text {bad }} \subseteq M$ such that

(1) the complement $M \backslash M^{\text {bad }}$ is in $\mathcal{C}^{\prime}$, and $G$ acts freely by orientation preserving automorphisms on it,

(2) there is a $G$-equivariant homeomorphism from $M_{0}^{\text {bad }}$ to $M^{\text {bad }}$,

(3) there is an isomorphism of $\mathbb{Q}[G]$-modules $H_{1}(M, \mathbb{Q}) \cong W$.

Remark 4.3. Condition (d) is automatically satisfied if $M_{0}^{\text {bad }}$ is a finite union of at most 1-dimensional submanifolds, possibly with boundary. In particular, such an $M_{0}^{\text {bad }} \subset M_{0}$ exists if $M_{0}$ is an oriented Riemannian orbifold, and $G$ acts effectively by orientation preserving isometries.

Theorem 4.2 essentially says that one cannot read off the geometry of the fixed point set in an orientation preserving $G$-action on a 3 -manifold $M$ from the $\mathbb{Q}[G]$-module structure of $H_{1}(M, \mathbb{Q})$. We now briefly contrast this with the situation in dimension 2 . We do not claim any originality in what follows, but we have not been able to find Corollary 4.6, in particular, stated in the literature.

The discussion will be most conveniently formulated in terms of characters, for which a general reference is [14. If $G$ is a finite group, and $U$ is a subgroup, we will denote by $\pi(U)$ the permutation character corresponding to the $G$-set $G / U$.

Theorem 4.4 (Artin's Induction Theorem). Let $G$ be a finite group. The $\mathbb{Q}$-vector space generated by the $\mathbb{Q}$-valued characters of $G$ is freely spanned by the permutation characters $\pi(C)$, as $C$ runs over $G$-conjugacy class representatives of cyclic subgroups of $G$.

Proof. See [14, Theorem 5.21],

The following result can be deduced from the Riemann-Hurwitz formula, and either the Lefschetz trace formula or Artin's Induction Theorem.

Proposition 4.5. Let $M$ be a closed connected orientable surface, let $G$ be a group of orientation preserving automorphisms of $M$, and let $\tau$ denote the genus of $M / G$. Let $\mathcal{S}$ be a full set of $G$-orbit representatives of the ramification points of the covering $M \rightarrow M / G$, and for each $P \in \mathcal{S}$, let $S_{P}$ be the stabiliser of $P$ in $G$. Let $\chi$ be the character corresponding to the $G$-module $H_{1}(M, \mathbb{Q})$. Then we have

$$
\chi=2 \pi(G)+(2 \tau-2+\# \mathcal{S}) \pi(\{1\})-\sum_{P \in \mathcal{S}} \pi\left(S_{P}\right) .
$$


Proof. See [5, Proposition 2].

It follows that, in the situation of Proposition 4.5, the structure of the ramification set of the covering $M \rightarrow M / G$ can be read off from the $\mathbb{Q}[G]$ module structure of $H_{1}(M, \mathbb{Q})$ in the following precise sense.

Corollary 4.6. Let $M$ and $M^{\prime}$ be closed connected orientable surfaces with an action of a finite group $G$ by orientation preserving automorphisms. If $P$ is a point on $M$ or $M^{\prime}$, let $S_{P}$ be its stabiliser in $G$. Suppose that the $\mathbb{Q}[G]$-modules $H_{1}(M, \mathbb{Q})$ and $H_{1}\left(M^{\prime}, \mathbb{Q}\right)$ are isomorphic. Then there exists a bijection $\beta$ between the ramification points of the cover $M \rightarrow M / G$, and those of the cover $M^{\prime} \rightarrow M^{\prime} / G$ such that for all ramification points $P \in M$, we have $S_{P}=S_{\beta(P)} \leq G$, so that, in particular, $\beta$ preserves ramification indices.

Proof. Let $\mathcal{S}$ and $\mathcal{S}^{\prime}$ be full sets of $G$-orbit representatives of the ramification points of $M \rightarrow M / G$, respectively of $M^{\prime} \rightarrow M^{\prime} / G$, and let $\tau$ and $\tau^{\prime}$ be the genera of $M / G$, respectively of $M^{\prime} / G$. By Proposition 4.5, there is an equality of characters

$$
(2 \tau-2+\# \mathcal{S}) \pi(\{1\})-\sum_{P \in \mathcal{S}} \pi\left(S_{P}\right)=\left(2 \tau^{\prime}-2+\# \mathcal{S}^{\prime}\right) \pi(\{1\})-\sum_{P^{\prime} \in \mathcal{S}^{\prime}} \pi\left(S_{P^{\prime}}\right) .
$$

Since none of the stabilisers $S_{P}$ and $S_{P^{\prime}}$ are trivial, and since they are all cyclic, it follows from Artin's Induction Theorem that there exists a bijection $\alpha$ from $\mathcal{S}$ to $\mathcal{S}^{\prime}$ such that for all $P \in \mathcal{S}$, we have $\pi\left(S_{P}\right)=\pi\left(S_{\alpha(P)}\right)$. This condition on the permutation characters is equivalent to $S_{P}$ being conjugate to $S_{\alpha(P)}$ in $G$. Since for every $P \in \mathcal{S}$, the set of stabilisers of the points in the $G$-orbit of $P$ is a single conjugacy class of subgroups, the result follows.

\section{Application to isospectral manifolds}

In this section we deduce Theorem 1.3 from Theorem 1.1. Our proof relies on Sunada's group theoretic construction of isospectral manifolds [23], and on the formalism of regulator constants, as introduced by DokchitserDokchitser, see e.g. [9].

5.1. Sunada's construction and the Cheeger-Müller theorem. If $p$ is a prime number, we will write $\mathbb{Z}_{(p)}$ for the localisation of $\mathbb{Z}$ at $p$, i.e. the subring $\left\{\frac{a}{b}: p \nmid b\right\}$ of $\mathbb{Q}$. In this subsection, $R$ will be either $\mathbb{Q}$ or $\mathbb{Z}_{(p)}$, where $p$ is a prime number.

Definition 5.1. Let $G$ be a finite group. An $R[G]$-relation is a formal linear combination $\sum_{i} U_{i}-\sum_{j} U_{j}^{\prime}$ of subgroups of $G$ with the property that there is an isomorphism of $R[G]$-modules

$$
\bigoplus_{i} R\left[G / U_{i}\right] \cong \bigoplus_{j} R\left[G / U_{j}^{\prime}\right]
$$

The following lemmas are routine, and we leave the proofs to the reader.

Lemma 5.2. If $G$ is a finite group, $N$ is a normal subgroup, and $\Theta=$ $\sum_{i} U_{i}-\sum_{j} U_{j}^{\prime}$ is an $R[G]$-relation, then $\operatorname{Def}_{G / N} \Theta=\sum_{i} N U_{i} / N-\sum_{j} N U_{j}^{\prime} / N$ is an $R[G / N]$-relation. 
Lemma 5.3. Let $G$ and $\tilde{G}$ be finite groups, let $U-U^{\prime}$ be an $R[G]$-relation, and let $\tilde{U}-\tilde{U}^{\prime}$ be an $R[\tilde{G}]$-relation. Then $U \times \tilde{U}-U^{\prime} \times \tilde{U}^{\prime}$ is a $R[G \times \tilde{G}]$ relation.

In [23. Sunada shows that $\mathbb{Q}[G]$-relations give rise to strongly isospectral manifolds, as follows.

Theorem 5.4 (Sunada, 23]). Let $G$ be a finite group, let $X \rightarrow Y$ be a $G$ covering of Riemannian manifolds, and let $U-U^{\prime}$ be a $\mathbb{Q}[G]$-relation. Then the intermediate coverings $X / U$ and $X / U^{\prime}$ are strongly isospectral.

It follows from the Cheeger-Müller Theorem [6, 18, 19] that if $M$ and $M^{\prime}$ are strongly isospectral Riemannian 3-manifolds, then

$$
\frac{\# H_{1}(M, \mathbb{Z})_{\text {tors }}}{\# H_{1}\left(M^{\prime}, \mathbb{Z}\right)_{\text {tors }}}=\frac{\operatorname{Reg}_{1}(M)^{2}}{\operatorname{Reg}_{1}\left(M^{\prime}\right)^{2}},
$$

where $\operatorname{Reg}_{1}(M)$ is the covolume of the lattice $H_{1}(M, \mathbb{Z}) / H_{1}(M, \mathbb{Z})_{\text {tors }}$ in the vector space $H_{1}(M, \mathbb{R})$ with respect to a certain canonical inner product, and similarly for $M^{\prime}$ - see [2] for details.

5.2. Regulator constants. When $M$ and $M^{\prime}$ arise from a $G$-covering $X \rightarrow$ $Y$ via Sunada's construction, we relate in [2] the regulator quotient of equation (5.5) to a certain representation theoretic invariant of $H_{1}(X, \mathbb{Q})$, called a regulator constant. We briefly recall the definition and some of the properties of this invariant. On the first reading, the definition may be skipped, since only the properties that we list below will be needed for the rest of the section.

Definition 5.6. Let $G$ be a finite group, let $\Theta=\sum_{i} U_{i}-\sum_{j} U_{j}^{\prime}$ be a $\mathbb{Q}[G]$ relation, and let $W$ be a finitely generated $\mathbb{Q}[G]$-module. Let $\langle$,$\rangle be a$ non-degenerate $G$-invariant $\mathbb{Q}$-bilinear pairing on $W$ with values in $\mathbb{Q}$. The regulator constant of $W$ with respect to $\Theta$ is defined as

$$
\mathcal{C}_{\Theta}(W)=\frac{\prod_{i} \operatorname{det}\left(\frac{1}{\# U_{i}}\langle,\rangle \mid W^{U_{i}}\right)}{\prod_{j} \operatorname{det}\left(\frac{1}{\# U_{j}^{\prime}}\langle,\rangle \mid W^{U_{j}^{\prime}}\right)} \in \mathbb{Q}^{\times} /\left(\mathbb{Q}^{\times}\right)^{2} .
$$

Here, each determinant is evaluated with respect to an arbitrary basis of the respective fixed space, and is therefore only well-defined modulo $\left(\mathbb{Q}^{\times}\right)^{2}$.

Remark 5.7. Let $G$ be a finite group, and let $W$ be a finitely generated $\mathbb{Q}[G]$-module. Choosing a pairing as in Definition 5.6 is equivalent to choosing an isomorphism of $\mathbb{Q}[G]$-modules between $W$ and its $\mathbb{Q}$-linear dual. Since finitely generated $\mathbb{Q}[G]$-modules are self-dual, such a pairing always exists.

Theorem 5.8. The value of $\mathcal{C}_{\Theta}(W)$ is independent of the pairing $\langle$,$\rangle .$

Proof. See [9, Theorem 2.17].

Theorem 5.8 justifies the notation $\mathcal{C}_{\Theta}(W)$, which makes no reference to the pairing. 
Example 5.9. Let $p$ be an odd prime number, and let $G_{p}=\mathrm{GL}_{2}\left(\mathbb{F}_{p}\right)$ be the group of invertible $2 \times 2$ matrices over the finite field with $p$ elements. Consider the following subgroups of $G_{p}$ :

$$
B_{p}=\left(\begin{array}{cc}
\mathbb{F}_{p}^{\times} & \mathbb{F}_{p} \\
0 & \mathbb{F}_{p}^{\times}
\end{array}\right), \quad U_{p}=\left(\begin{array}{cc}
\left(\mathbb{F}_{p}^{\times}\right)^{2} & \mathbb{F}_{p} \\
0 & \mathbb{F}_{p}^{\times}
\end{array}\right), \quad U_{p}^{\prime}=\left(\begin{array}{cc}
\mathbb{F}_{p}^{\times} & \mathbb{F}_{p} \\
0 & \left(\mathbb{F}_{p}^{\times}\right)^{2}
\end{array}\right) .
$$

The permutation module $\mathbb{Q}\left[G_{p} / U_{p}\right]$ decomposes as a direct sum $\mathbb{Q}\left[G_{p} / U_{p}\right] \cong$ $\mathbb{Q}\left[G_{p} / B_{p}\right] \oplus I_{p}$, where $I_{p}$ is a simple $\mathbb{Q}\left[G_{p}\right]$-module of dimension $(p+1)$ over $\mathbb{Q}$. Moreover, the formal linear combination $\Theta=U_{p}-U_{p}^{\prime}$ is a $\mathbb{Q}\left[G_{p}\right]$ relation, and for every prime number $q \neq p$, it is a $\mathbb{Z}_{(q)}\left[G_{p}\right]$-relation. In [2, Proposition 4.2] we showed that $\mathcal{C}_{\Theta}\left(I_{p}\right) \equiv p\left(\bmod \left(\mathbb{Q}^{\times}\right)^{2}\right)$.

Example 5.10. Let $G_{2}$ be the affine linear group over $\mathbb{Z} / 8 \mathbb{Z}$, i.e. the group of linear transformations $T_{a, b}: x \mapsto a x+b$ of $\mathbb{Z} / 8 \mathbb{Z}$, where $a \in(\mathbb{Z} / 8 \mathbb{Z})^{\times}$ and $b \in \mathbb{Z} / 8 \mathbb{Z}$. Consider the following subgroups of $G_{2}$ :

$$
\begin{aligned}
U_{2} & =\left\langle T_{a, 0}: a \in(\mathbb{Z} / 8 \mathbb{Z})^{\times}\right\rangle, \\
U_{2}^{\prime} & =\left\langle T_{3,4}, T_{-1,0}\right\rangle, \\
B_{2} & =\left\langle T_{3,4}, T_{a, 0}: a \in(\mathbb{Z} / 8 \mathbb{Z})^{\times}\right\rangle .
\end{aligned}
$$

The group $G_{2}$ is isomorphic to the semidirect product $\mathbb{Z} / 8 \mathbb{Z} \rtimes(\mathbb{Z} / 8 \mathbb{Z})^{\times}$; the subgroups $U_{2}$ and $U_{2}^{\prime}$ are both isomorphic to $C_{2} \times C_{2} ; \Theta=U_{2}-U_{2}^{\prime}$ is a $\mathbb{Q}\left[G_{2}\right]$-relation, and for every odd prime number $q$, it is a $\mathbb{Z}_{(q)}\left[G_{2}\right]$-relation. Moreover, $\mathbb{Q}\left[G_{2} / U_{2}\right]$ decomposes as a direct sum $\mathbb{Q}\left[G_{2} / U_{2}\right] \cong \mathbb{Q}\left[G_{2} / B_{2}\right] \oplus I_{2}$, where $I_{2}$ is a simple $\mathbb{Q}\left[G_{2}\right]$-module of dimension 4 over $\mathbb{Q}$, and one can show by a direct computation that $\mathcal{C}_{\Theta}\left(I_{2}\right) \equiv 2\left(\bmod \left(\mathbb{Q}^{\times}\right)^{2}\right)$.

Regulator constants satisfy the following properties:

(Reg 1) if $G$ is a finite group, $N$ is a normal subgroup, $\Theta$ is a $\mathbb{Q}[G]$-relation, $W$ is a $\mathbb{Q}[G / N]$-module, and $\operatorname{Inf}_{G / N} W$ is the lift of $W$ to a $\mathbb{Q}[G]$ module, then $\mathcal{C}_{\Theta}\left(\operatorname{Inf}_{G / N} W\right)=\mathcal{C}_{\operatorname{Def}_{G / N} \Theta}(W)$;

(Reg 2) if $G$ is a finite group, $\Theta$ is a $\mathbb{Q}[G]$-relation, and $W_{1}, W_{2}$ are $\mathbb{Q}[G]$ modules, then $\mathcal{C}_{\Theta}\left(W_{1} \oplus W_{2}\right)=\mathcal{C}_{\Theta}\left(W_{1}\right) \cdot \mathcal{C}_{\Theta}\left(W_{2}\right)$.

Lemma 5.11. Let $\mathcal{P}$ be a finite set of prime numbers. Then there exist a finite group $G, a \mathbb{Q}[G]$-relation $\Theta=U-U^{\prime}$, and a $\mathbb{Q}[G]$-module $W$, such that

(1) we have

$$
\mathcal{C}_{\Theta}(W) \equiv \prod_{p \in \mathcal{P}} p \quad\left(\bmod \left(\mathbb{Q}^{\times}\right)^{2}\right)
$$

(2) for all prime numbers $q \notin \mathcal{P}$, the relation $\Theta$ is a $\mathbb{Z}_{(q)}[G]$-relation.

Proof. Let $G=\prod_{p \in \mathcal{P}} G_{p}$, where $G_{p}$ is as in Example 5.9 when $p$ is odd, and as in Example 5.10 when $p=2$. For each $p \in \mathcal{P}$, let $\bar{N}_{p}$ denote the kernel of the projection map $G \rightarrow G_{p}$, so that the quotient $G / N_{p}$ is isomorphic to $G_{p}$.

We may lift the module $I_{p}$ of Example 5.9 , respectively 5.10 from $G / N_{p}$ to a $\mathbb{Q}[G]$-module $\operatorname{Inf}_{G / N_{p}} I_{p}$. Let $W$ be the direct sum of $\mathbb{Q}[G]$-modules $W=\bigoplus_{p \in \mathcal{P}} \operatorname{Inf}_{G / N_{p}} I_{p}$. Let $U=\prod_{p \in \mathcal{P}} U_{p} \leq G$, where the subgroups $U_{p} \leq$ $G_{p}$ are as in Example 5.9, respectively 5.10, and define $U^{\prime}$ analogously. So for every $p \in \mathcal{P}$, the image of $U$ under the quotient map $G \rightarrow G / N_{p}$ is $U_{p}$, and the image of $U^{\prime}$ is $U_{p}^{\prime}$. 
By Lemma 5.3, the formal linear combination $\Theta=U-U^{\prime}$ is a $\mathbb{Q}[G]$ relation, and for every prime number $q \notin \mathcal{P}$, it is also a $\mathbb{Z}_{(q)}[G]$-relation. This proves the second part of the lemma.

By property (Reg 2), property (Reg 1), and Examples 5.9 and 5.10, in that order, we have

$$
\begin{aligned}
\mathcal{C}_{\Theta}(W) & \equiv \prod_{p \in \mathcal{P}} \mathcal{C}_{\Theta}\left(\operatorname{Inf}_{G / N_{p}} I_{p}\right) \equiv \prod_{p \in \mathcal{P}} \mathcal{C}_{\operatorname{Def}_{G / N_{p}} \Theta}\left(I_{p}\right) \\
& \equiv \prod_{p \in \mathcal{P}} p \quad\left(\bmod \left(\mathbb{Q}^{\times}\right)^{2}\right),
\end{aligned}
$$

which proves the first part of the lemma.

5.3. Isospectral manifolds. The following two results are the crucial ingredients that will allow us to deduce Theorem 1.3 from Theorem 1.1 .

Proposition 5.12. Let $G$ be a finite group, let $X \rightarrow Y$ be a $G$-covering of Riemannian manifolds, and let $\Theta=U-U^{\prime}$ be a $\mathbb{Q}[G]$-relation. Then $\frac{\operatorname{Reg}_{1}(X / U)^{2}}{\operatorname{Reg}_{1}\left(X / U^{\prime}\right)^{2}} \in \mathbb{Q}^{\times}$, and we have

$$
\frac{\operatorname{Reg}_{1}(X / U)^{2}}{\operatorname{Reg}_{1}\left(X / U^{\prime}\right)^{2}} \equiv \mathcal{C}_{\Theta}\left(H_{1}(X, \mathbb{Q})\right) \quad\left(\bmod \left(\mathbb{Q}^{\times}\right)^{2}\right) .
$$

Proof. This is a special case of [2, Corollary 3.12].

Proposition 5.13. Let $G$ be a finite group, let $X \rightarrow Y$ be a $G$-covering of Riemannian manifolds, let $q$ be a prime number, and let $\Theta=U-U^{\prime}$ be a $\mathbb{Z}_{(q)}[G]$-relation. Then we have

$$
H_{1}(X / U, \mathbb{Z})\left[q^{\infty}\right] \cong H_{1}\left(X / U^{\prime}, \mathbb{Z}\right)\left[q^{\infty}\right] .
$$

Proof. This is a special case of [2, Theorem 3.5].

We can now prove Theorem 1.3 . We recall the statement.

Theorem 5.14. Let $\mathcal{P}$ be a finite set of prime numbers. Then there exist closed connected orientable 3-manifolds $M$ and $M^{\prime}$ that are strongly isospectral with respect to hyperbolic metrics and such that

(1) for all $p \in \mathcal{P}$ we have

$$
\# H_{1}(M, \mathbb{Z})\left[p^{\infty}\right] \neq \# H_{1}\left(M^{\prime}, \mathbb{Z}\right)\left[p^{\infty}\right] ;
$$

(2) for all prime numbers $q \notin \mathcal{P}$ we have an isomorphism of Abelian groups

$$
H_{1}(M, \mathbb{Z})\left[q^{\infty}\right] \cong H_{1}\left(M^{\prime}, \mathbb{Z}\right)\left[q^{\infty}\right] .
$$

Proof. Let $G, U, U^{\prime}, \Theta$, and $W$ be as in Lemma 5.11 applied to the set $\mathcal{P}$. By Theorem 1.1, there exists a closed hyperbolic 3-manifold $X$ with a free $G$-action such that there is an isomorphism of $\mathbb{Q}[G]$-modules $H_{1}(X, \mathbb{Q}) \cong$ $W$. Let $M=X / U$ and $M^{\prime}=X / U^{\prime}$. The second part of the theorem immediately follows from Lemma 5.11 (2) and Proposition 5.13 
To prove the first part, we invoke equation (5.5), Proposition 5.12, and Lemma 5.11 (1), in that order, to conclude that

$$
\begin{aligned}
\frac{\# H_{1}(M, \mathbb{Z})_{\text {tors }}}{\# H_{1}\left(M^{\prime}, \mathbb{Z}\right)_{\text {tors }}} & =\frac{\operatorname{Reg}_{1}(M)^{2}}{\operatorname{Reg}_{1}\left(M^{\prime}\right)^{2}} \\
& \equiv \mathcal{C}_{\Theta}\left(H_{1}(X, \mathbb{Q})\right)=\mathcal{C}_{\Theta}(W) \\
& \equiv \prod_{i} p_{i}\left(\bmod \left(\mathbb{Q}^{\times}\right)^{2}\right),
\end{aligned}
$$

which completes the proof.

\section{REFERENCES}

[1] D. Auckly, H. J. Kim, P. Melvin, and D. Ruberman, Equivariant hyperbolization of 3-manifolds via homology cobordisms, arXiv:1804.03777v1 [math.GT] (2018).

[2] A. Bartel and A. Page, Torsion homology and regulators of isospectral manifolds, J. Topology 9 no. 4 (2016), 1237-1256.

[3] M. Boileau and S. Wang, Non-zero degree maps and surface bundles over $S^{1}$, J. Differential Geom. 43 no. 4 (1996), 789-806.

[4] W. Bosma and B. de Smit, On arithmetically equivalent number fields of small degree, Algorithmic number theory (Sydney, 2002), 67-79, Lecture Notes in Comput. Sci. 2369, Springer (2002).

[5] S. A. Broughton, The Homology and Higher Representations of the Automorphism Group of a Riemann Surface, Trans. Amer. Math. Soc. 300 no. 1 (1987), 153-158.

[6] J. Cheeger, Analytic torsion and the heat equation, Ann. of Math. 109 (1979), 259322.

[7] D. Cooper and D. D. Long, Free actions of finite groups on rational homology 3spheres, Topology Appl. 101 no. 2 (2000), 143-148.

[8] B. de Smit and R. Perlis, Zeta functions do not determine class numbers, Bull. Amer. Math. Soc. 31 no. 2 (1994), 213-215.

[9] T. Dokchitser and V. Dokchitser, Regulator constants and the parity conjecture, Invent. Math. 178 no. 1 (2009), 23-71.

[10] A. Edmonds, Aspects of group actions on four manifolds, Topology Appl. 31 no. 2 (1989), 109-124

[11] C. Gordon, Survey of isospectral manifolds. Handbook of differential geometry Vol. I (2000), 747-778.

[12] C. Gordon, P. Perry and D. Schueth, Isospectral and isoscattering manifolds: a survey of techniques and examples, Geometry, spectral theory, groups, and dynamics, 157179, Contemp. Math. 387, Amer. Math. Soc. (2005).

[13] A. Ikeda, On the spectrum of a Riemannian manifold of positive constant curvature, Osaka J. Math. 17 no. 1 (1980), 75-93.

[14] M. Isaacs, Character Theory of Finite Groups, Academic Press (1976).

[15] M. A. Kervaire and J. W. Milnor, Groups of homotory Spheres: I, Annals of Math. 77 no. 3 (1963), 504-537.

[16] T. Y. Lam, A First Course in Noncommutative Rings, GTM 131, Springer (2001).

[17] E. Lauret, R. Miatello and J. P. Rossetti, Strongly isospectral manifolds with nonisomorphic cohomology rings, Rev. Mat. Iberoam. 29 (2013), 611-634.

[18] W. Müller, Analytic torsion and $R$-torsion of Riemannian manifolds, Adv. Math. 28 (1978), 233-305.

[19] W. Müller, Analytic torsion and $R$-torsion for unimodular representations, J. Amer. Math. Soc. 6 (1993), 721-753.

[20] W. Scharlau, Quadratic and Hermitian forms, Grundlehren der Mathematischen Wissenschaften 270, Springer (1985).

[21] D. Schueth, Isospectral, non-isometric Riemannian manifolds, Geometry and physics (Zdkov, 1993), Rend. Circ. Mat. Palermo (2) Suppl. No. 37 (1994), 207-231.

[22] A. Sikora, Analogies between group actions on 3-manifolds and number fields, Comment. Math. Helv. 78 no. 4 (2003), 832-844. 
[23] T. Sunada, Riemannian coverings and isospectral manifolds, Annals Math. 121 no. 1 (1985), 169-186.

[24] W. P. Thurston, The Geometry and Topology of 3-Manifolds, Electronic version 1.1 (2002).

School of Mathematics and Statistics, University of Glasgow, Glasgow G12 8QQ, Scotland, UK

InRIA, Univ. Bordeaux, CNRS, IMB, UMR5251, F-33400 Talence, France

E-mail address: alex.bartel@glasgow.ac.uk, aurel.page@inria.fr 